PROCEEDINGS OF THE

AMERICAN MATHEMATICAL SOCIETY

Volume 133, Number 2, Pages 617-625

S 0002-9939(04)07543-4

Article electronically published on August 20, 2004

\title{
RATIONAL IRREDUCIBLE PLANE CONTINUA WITHOUT THE FIXED-POINT PROPERTY
}

\author{
CHARLES L. HAGOPIAN AND ROMAN MAŃKA
}

(Communicated by Alan Dow)

\begin{abstract}
We define rational irreducible continua in the plane that admit fixed-point-free maps with the condition that all of their tranches have the fixed-point property. This answers in the affirmative a question of Hagopian. The construction is based on a special class of spirals that limit on a double Warsaw circle. The closure of each of these spirals has the fixed-point property.
\end{abstract}

\section{INTRODUCTION}

A continuum is a non-void compact connected metric space. A continuum is irreducible between two of its points if none of its proper subcontinua contains both of these points. A continuum is rational if each of its points admits a base of neighborhoods with countable boundaries.

Since rational continua are hereditarily decomposable [Ku2, Thm. 5, p. 285], each rational irreducible continuum $C$ is of type $\lambda$, i.e., of the order type of the unit interval $[0,1]$. This means, following Knaster and Kuratowski Ku1, §3, pp. $248,262]$, that $C$ admits a uniquely determined monotone upper semi-continuous decomposition to an arc (i.e., homeomorphic image of $[0,1]$ ) with the property that each element of the decomposition has void interior relative to $C \mathrm{Ku} 2$, Thm. 3, p. 216, Thm. 2, p. 215]. The continua that are elements of this decomposition are called the tranches of $C$.

A continuum has the fixed-point property if each map of it into itself has a fixed point.

To answer a question of Gordh [L, Prob. 43, p. 371], Hagopian [H] recently defined a non-planar irreducible rational continuum $\mathcal{M}$ such that each tranche of $\mathcal{M}$ has the fixed-point property and $\mathcal{M}$ admits a fixed-point-free map. Hagopian [H, Ques. 1] asked if there is a continuum with these properties in the plane. Here we give a positive answer to this question.

A double Warsaw circle is a union of two disjoint rays each of which limits on an initial arc of the other. It follows from a result of Nadler [N, Lem. 11, p. 131] that each double Warsaw circle is planar (also see Awartani's theorem [A, Thm. 4.1, p. 232]).

Received by the editors March 13, 2003 and, in revised form, October 17, 2003

2000 Mathematics Subject Classification. Primary 54F15, 54H25.

Key words and phrases. Fixed-point property, rational continua, irreducible continua of type $\lambda$, spiral, double Warsaw circle, plane continua, retractions.

The authors wish to thank Mark Marsh for helpful conversations about the topics of this paper.

(C)2004 American Mathematical Society 
Our construction involves two spirals limiting on a double Warsaw circle. One spiral lies outside and the other inside of the double Warsaw circle. The closure of one spiral has the fixed-point property, and the other does not. The closure that has the fixed-point property is approximated by a ray in such a way that the resulting irreducible rational continuum can be retracted onto the closure of the spiral without the fixed-point property.

\section{Preliminaries on RAYS AND ARCS}

In this paper a ray is a topological half-line, i.e., homeomorphic image of $[0,1)$. Every ray $R$ will be considered with its natural order $<_{R}$ inherited from $[0,1)$.

If $R$ is a ray contained in a continuum, then $C l R$ (the closure of $R$ ) is a continuum irreducible between the origin (initial point) of $R$ and each limit point of $R$, i.e., a limit of an increasing convergent sequence of points of $R$ [M2, Prop. 2.1]. The continuum $\mathrm{ClR}$ is rational if (and only if) the continuum $L(R)$ consisting of all limit points of $R$ is rational. Obviously, $R \cap L(R)=\emptyset$ and $R \cup L(R)=C l R$. A continuum $C$ of the form $C=C l R \cup C l S$, where $R$ and $S$ are rays with origins $p$ and $q$, respectively, is irreducible between $p$ and $q$ if $R \cap C l S=\emptyset=S \cap C l R$ (see [M1 Rem. 1, p. 125] for a general statement).

A space is (uniquely) arcwise connected if every two of its points are end points of a (unique) arc in the space.

Lemma 2.1. Suppose $f$ is a map of a space $L$ into a uniquely arcwise connected space and an arc $M$ is contained in $f(L)$. Then for every family of arcwise connected sets $L_{1}, L_{2}, \cdots, L_{v}$ such that $L=\bigcup_{j=1}^{v} L_{j}$ and for every partition $\mathcal{P}(M)$ of $M$ into consecutive arcs $M_{1}, M_{2}, \cdots, M_{\mu}$ with $v \leq \mu$, there exist $L_{j}$ and $M_{i}$ such that $M_{i} \subset f\left(L_{j}\right)$.

Proof. Let $q_{0}, q_{1}, \cdots, q_{\mu}$ be the consecutive end points of the $\operatorname{arcs} M_{1}, M_{2}, \cdots, M_{\mu}$ belonging to $\mathcal{P}(M)$. We have $M_{i} \cap M_{i+1}=\left\{q_{i}\right\}$ for each $i=1,2, \cdots, \mu-1$. For each $j=1,2, \cdots, v$, let $\Sigma_{j}=\left\{i \mid q_{i} \in f\left(L_{j}\right)\right\}$. It suffices to show that some $\Sigma_{j}$ has more than one element.

Suppose, on the contrary, that each $\Sigma_{j}$ has at most one element. Then the set $\Sigma=\bigcup_{j=1}^{v} \Sigma_{j}$ has at most $v$ elements. Since $M \subset f(L)=f\left(\bigcup_{j=1}^{v} L_{j}\right)$, it follows that $\Sigma=\left\{i \mid q_{i} \in f(L)\right\}$ and $\{0,1,2, \cdots, \mu\} \subset \Sigma$. Hence $\mu+1 \leq v$. This contradicts the assumption that $v \leq \mu$.

This lemma will be used in Section 5. Given a map $f$, we will say sometimes that $X$ is mapped on $Y$ when $Y \subset f(X)$.

Notation. For each two points $a$ and $b$ of the plane $E^{2}$, we denote the straight line interval with end points $a$ and $b$ by $\overline{a b}$. If $a$ and $b$ belong to a uniquely arcwise connected set $A$, then $A[a, b]$ will denote the unique arc in $A$ with end points $a$ and $b$. Given a set $Z$ in $E^{2}$ and a real number $\epsilon$, we let $Z \uparrow \epsilon$ denote $\left\{(x, y) \in E^{2} \mid(x, y-\epsilon) \in Z\right\}$. We denote the set of all positive integers $1,2, \cdots$ by $N$.

\section{A Class of spirals Limiting on a double WarsaW CirCle}

Let $\gamma$ be the map of $(0,1]$ onto $[1,3]$ defined by $\gamma(x)=2+\sin \frac{\pi}{x}$, and let $\Gamma$ denote the graph of $\gamma$ in $E^{2}$, i.e., $\Gamma=\left\{(x, \gamma(x)) \in E^{2} \mid x \in(0,1]\right\}$. 
The set $A=\overline{(0,-1)(0,-3)} \cup \overline{(0,-3)(2,0)} \cup \overline{(2,0)(1,2)} \cup \Gamma$ is a ray with origin $(0,-1)$ that limits on the interval $\overline{(0,1)(0,3)}$. Note that $C l A$ is the topologist's sine curve.

Let $\alpha$ be the map of $E^{2}$ onto $E^{2}$ defined by $\alpha(x, y)=(-x,-y)$.

Let $D$ be the continuum $A \cup \alpha(A)$ in $E^{2}$. Note that $D$ is a double Warsaw circle.

Consider two sequences $\left\{k_{n}\right\}_{n \in N}$ and $\left\{l_{n}\right\}_{n \in N}$ of positive integers satisfying the following conditions:

(3.1). $\quad k_{1} \leq k_{2} \leq \cdots \leq k_{n} \leq k_{n+1} \leq \cdots \rightarrow \infty$ and $l_{1} \leq l_{2} \leq \cdots \leq l_{n} \leq l_{n+1} \leq$ $\cdots \rightarrow \infty$.

Similar to $T$ in Example 4.1 of $[\mathrm{H}-\mathrm{M}]$, we define a spiral $S\left\{k_{n}, l_{n}\right\}_{n \in N}$ limiting on $D$.

For each positive integer $n$, let $\mu_{n}=\frac{2}{4 n+1}$ and let $p_{n}$ be the point $\left(\mu_{n}, 3\right)$ of $\Gamma$.

For each $n$, let

$$
\begin{aligned}
& A_{n}=\overline{\left(2+n^{-1}, 0\right)\left(1,2+n^{-1}\right)}, \\
& B_{n}\left(k_{n}\right)=A\left[(1,2), p_{k_{n}}\right] \uparrow n^{-1}, \\
& C_{n}\left(k_{n}\right)=\left\{(x, y) \in E^{2} \mid 0 \leq x \leq \mu_{k_{n}} \text { and } y=3+n^{-1}\right\}, \\
& D_{n}=\overline{\left(0,3+n^{-1}\right)\left(-2-n^{-1}, 0\right)}, \\
& E_{n}\left(l_{n}\right)=\alpha\left(A\left[(1,2), p_{l_{n}}\right]\right) \uparrow-n^{-1}, \\
& F_{n}\left(l_{n}\right)=\left\{(x, y) \in E^{2} \mid-\mu_{l_{n}} \leq x \leq 0 \text { and } y=-3-n^{-1}\right\}, \text { and } \\
& G_{n}=\overline{\left(0,-3-n^{-1}\right)\left(2+(n+1)^{-1}, 0\right)} .
\end{aligned}
$$

For each $n$, let

$$
\Gamma_{n}\left(k_{n}, l_{n}\right)=A_{n} \cup B_{n}\left(k_{n}\right) \cup C_{n}\left(k_{n}\right) \cup D_{n} \cup \alpha\left(A_{n}\right) \cup E_{n}\left(l_{n}\right) \cup F_{n}\left(l_{n}\right) \cup G_{n} .
$$

Let

$$
S\left\{k_{n}, l_{n}\right\}_{n \in N}=\bigcup_{n=1}^{\infty} \Gamma_{n}\left(k_{n}, l_{n}\right) .
$$

Note that $S\left\{k_{n}, l_{n}\right\}_{n \in N}$ is a ray with origin $(3,0)$ that limits on the double Warsaw circle $D$.

Let $X$ be an arc in $S\left\{k_{n}, l_{n}\right\}_{n \in N}$ that is contained in $E_{+}^{2}=\left\{(x, y) \in E^{2} \mid y>0\right\}$ or $E_{-}^{2}=\left\{(x, y) \in E^{2} \mid y<0\right\}$. A subarc $Y$ of $X$ is called a dip (hump) in $X$ if the end points of $Y$ are relative maximum (minimum) points of $X$ and no interior point of $Y$ is a relative maximum (minimum) of $X$. Note that the $n$-th component of $S\left\{k_{n}, l_{n}\right\}_{n \in N} \cap E_{+}^{2}$ has $k_{n}$ dips, all contained in $B_{n}\left(k_{n}\right)$, and the $n$-th component of $S\left\{k_{n}, l_{n}\right\}_{n \in N} \cap E_{-}^{2}$ has $l_{n}$ humps, all contained in $E_{n}\left(l_{n}\right)$.

Proposition 3.2. The continuum $D \cup S\{2 n-1,2 n\}_{n \in N}$ does not have the fixedpoint property.

The proof of Proposition 3.2 is given in Examples 4.1 and 4.2 of $[\mathrm{H}-\mathrm{M}]$.

Theorem 3.3. If one of the sequences $\left\{\frac{l_{n}-k_{n}}{k_{n}}\right\}_{n \in N}$ or $\left\{\frac{k_{n+1}-l_{n}}{l_{n}}\right\}_{n \in N}$ is unbounded, then the continuum $D \cup S\left\{k_{n}, l_{n}\right\}_{n \in N}$ has the fixed-point property.

The proof of Theorem 3.3 will be provided in Section 5. Here, let us comment upon the assumption of Theorem 3.3.

Remark 3.4. If $k_{1} \leq l_{1} \leq k_{2} \leq l_{2} \leq \cdots \leq k_{n} \leq l_{n} \leq k_{n+1} \leq \cdots \rightarrow \infty$, then the assumption of Theorem 3.3 is equivalent to each of the following two conditions:

(1) one of the sequences $\left\{\frac{k_{n+1}}{k_{n}}\right\}_{n \in N}$ or $\left\{\frac{l_{n+1}}{l_{n}}\right\}_{n \in N}$ is unbounded; 
(2) both of the sequences $\left\{\frac{k_{n+1}}{k_{n}}\right\}_{n \in N}$ and $\left\{\frac{l_{n+1}}{l_{n}}\right\}_{n \in N}$ are unbounded.

Indeed, for each positive integer $n$, setting $s_{n}=\frac{l_{n}-k_{n}}{k_{n}}$ and $t_{n}=\frac{k_{n+1}-l_{n}}{l_{n}}$, we have $l_{n}-k_{n}=s_{n} k_{n}$ and $k_{n+1}-l_{n}=t_{n} l_{n}$. Hence $l_{n}=\left(s_{n}+1\right) k_{n}$ and $k_{n+1}=\left(t_{n}+1\right) k_{n}$, and therefore $k_{n+1}-k_{n}=\left(k_{n+1}-l_{n}\right)+\left(l_{n}-k_{n}\right)=t_{n} l_{n}+s_{n} k_{n}=t_{n}\left(s_{n}+1\right) k_{n}+$ $s_{n} k_{n}=\left(s_{n}+s_{n} t_{n}+t_{n}\right) k_{n}$. Thus $\frac{k_{n+1}}{k_{n}}=s_{n}+s_{n} t_{n}+t_{n}+1$. By an analogous calculation, $\frac{l_{n+1}}{l_{n}}=s_{n+1}+s_{n+1} t_{n}+t_{n}+1$.

For each $n$, let $v_{n}=\frac{2}{4 n-1}$ and let $q_{n}$ be the point $\left(v_{n}, 1\right)$ of $\Gamma$.

Proposition 3.5. There exist a ray $S^{o}\{2 n-1,2 n\}_{n \in N}$ in $E^{2}$ inside of the double Warsaw circle $D$ and a homeomorphism $h_{0}$ of $D \cup S\{2 n-1,2 n\}_{n \in N}$ onto $D \cup$ $S^{o}\{2 n-1,2 n\}_{n \in N}$ that is the identity on $D$.

Proof. For each positive integer $n$, let

$$
\begin{gathered}
A_{n}^{o}=\overline{\left(2-(n+1)^{-1}, 0\right)\left(1,2-(n+1)^{-1}\right)} \\
B_{n}^{o}(2 n-1)=A\left[(1,2), q_{2 n-1} \uparrow-(n+1)^{-1},\right. \\
C_{n}^{o}(2 n-1)=\left\{(x, y) \in E^{2} \mid-(n+1)^{-1} \leq x \leq v_{2 n-1} \text { and } y=1-(n+1)^{-1}\right\} \\
\cup \overline{\left(-(n+1)^{-1}, 1-(n+1)^{-1}\right)\left(-(n+1)^{-1}, 3-(n+1)^{-1}\right)}, \\
D_{n}^{o}=\overline{\left(-(n+1)^{-1}, 3-(n+1)^{-1}\right)\left(2-(n+1)^{-1}, 0\right)}, \\
E_{n}^{o}(2 n)=\left\{(2 n)=\alpha\left(A\left[(1,2), q_{2 n}\right]\right) \uparrow(n+1)^{-1},\right. \\
\cup \overline{\left((n+1)^{-1},-1+(n+1)^{-1}\right)\left((n+1)^{-1},-3+(n+1)^{-1}\right)}, \\
G_{n}^{o}=\overline{\left((n+1)^{-1},-3+(n+1)^{-1}\right)\left(2-(n+2)^{-1}, 0\right)} .
\end{gathered}
$$

Observe that $B_{n}^{o}(2 n-1)$ has $2 n-2$ dips and $E_{n}^{o}(2 n)$ has $2 n-1$ humps.

For each $n \in N$, let

$\Gamma_{n}^{o}(2 n-1,2 n)=A_{n}^{o} \cup B_{n}^{o}(2 n-1) \cup C_{n}^{o}(2 n-1) \cup D_{n}^{o} \cup \alpha\left(A_{n}^{o}\right) \cup E_{n}^{o}(2 n) \cup F_{n}^{o}(2 n) \cup G_{n}^{o}$.

Define

$$
S^{o}\{2 n-1,2 n\}_{n \in N}=\bigcup_{n=1}^{\infty} \Gamma_{n}^{o}(2 n-1,2 n) .
$$

Note that $S^{o}\{2 n-1,2 n\}_{n \in N}$ is a ray with origin $\left(\frac{3}{2}, 0\right)$ that limits on the double Warsaw circle $D$.

For each $n \in N$, let $h_{0}$ send the $\operatorname{arcs} A_{n}, B_{n}(2 n-1) \cup C_{n}(2 n-1), D_{n}, \alpha\left(A_{n}\right)$, $E_{n}(2 n) \cup F_{n}(2 n)$, and $G_{n}$ onto the corresponding $\operatorname{arcs} A_{n}^{o}, B_{n}^{o}(2 n-1) \cup C_{n}^{o}(2 n-1)$, $D_{n}^{o}, \alpha\left(A_{n}^{o}\right), E_{n}^{o}(2 n) \cup F_{n}^{o}(2 n)$, and $G_{n}^{o}$. The map $h_{0}$ is affine and order-preserving on each arc. Observe that $h_{0}$ sends the final dip of $B_{n}(2 n-1) \cup C_{n}(2 n-1)$ onto the arc $B_{n}^{o}(2 n-1)\left[\left(\mu_{2 n-1}, 3-(n+1)^{-1}\right)\left(v_{2 n-1}, 1-(n+1)^{-1}\right)\right] \cup C_{n}^{o}(2 n-1)$, which indeed is a dip. Also $h_{0}$ sends the final hump of $E_{n}(2 n) \cup F_{n}(2 n)$ onto the arc $E_{n}^{o}(2 n)\left[\left(-\mu_{2 n},-3+(n+1)^{-1}\right)\left(-v_{2 n},-1+(n+1)^{-1}\right)\right] \cup F_{n}^{o}(2 n)$, which is a hump. The map $h_{0}$ is a one-to-one continuous extension of the identity on $D$.

Proposition 3.6. If $2 n-1 \leq k_{n}$ and $2 n \leq l_{n}$ for each $n \in N$, then there is a map $\rho_{1}$ of $D \cup S\left\{k_{n}, l_{n}\right\}_{n \in N}$ onto $D \cup S\{2 n-1,2 n\}_{n \in N}$ that is the identity on $D$.

Proof. Observe that among the $\operatorname{arcs} A_{n}, \cdots, G_{n}$ that define $S\{2 n-1,2 n\}_{n \in N}$ or $S\left\{k_{n}, l_{n}\right\}_{n \in N}$ only the arcs $B_{n}(2 n-1), C_{n}(2 n-1), E_{n}(2 n)$, and $F_{n}(2 n)$ may differ from $B_{n}\left(k_{n}\right), C_{n}\left(k_{n}\right), E_{n}\left(l_{n}\right)$, and $F_{n}\left(l_{n}\right)$, respectively. Outside of these arcs, let 
$\rho_{1}$ be the identity. Also on $B_{n}\left(k_{n}\right) \cup C_{n}\left(k_{n}\right)$ (respectively, $E_{n}\left(l_{n}\right) \cup F_{n}\left(l_{n}\right)$ ), let $\rho_{1}$ be the identity on the first $2 n-1$ dips (respectively, $2 n$ humps). Let $\rho_{1}$ collapse the remaining $k_{n}-(2 n-1)$ dips (respectively, $l_{n}-2 n$ humps) onto the subarc of the arc $B_{n}(2 n-1)$ (respectively, $\left.E_{n}(2 n)\right)$ that has end points $\left(\mu_{2 n-1}, 3+n^{-1}\right)$ and $\left(v_{2 n-1}, 1+n^{-1}\right)$ (respectively, $\left(-\mu_{2 n},-3-n^{-1}\right)$ and $\left(-v_{2 n},-1-n^{-1}\right)$. Simultaneously, $\rho_{1}$ sends the $\operatorname{arc} C_{n}\left(k_{n}\right)$ onto $C_{n}(2 n-1)$, and $F_{n}\left(l_{n}\right)$ onto $F_{n}(2 n)$.

\section{Main continuum $\mathcal{M}_{0}$}

Suppose $\left\{k_{n}, l_{n}\right\}_{n \in N}$ satisfies the assumptions of Theorem 3.3 and Proposition 3.6, that is, (1) $\left\{k_{n}\right\}_{n \in N}$ and $\left\{l_{n}\right\}_{n \in N}$ are sequences of positive integers satisfying (3.1), (2) $\left\{\frac{l_{n}-k_{n}}{k_{n}}\right\}_{n \in N}$ or $\left\{\frac{k_{n+1}-l_{n}}{l_{n}}\right\}_{n \in N}$ is unbounded, and (3) $2 n-1 \leq k_{n}$ and $2 n \leq l_{n}$ for each $n \in N$. Let $R$ be a ray in the unbounded domain of $E^{2}-D$, with origin $(4,0)$, consisting of folded arcs of increasing lengths that limit on $D \cup S\left\{k_{n}, l_{n}\right\}_{n \in N}$ (taking a pattern by the graph of the map $g$ of $(0,1]$ onto $[0, \infty)$ given by $g(x)=\frac{1}{x}\left(1+\sin \frac{\pi}{x}\right)$, with $(0,0)$ corresponding to the origin $(3,0)$ of $\left.S\left\{k_{n}, l_{n}\right\}_{n \in N}\right)$. Let $\rho_{2}$ be the natural retraction of $D \cup S\left\{k_{n}, l_{n}\right\}_{n \in N} \cup R$ onto $D \cup S\left\{k_{n}, l_{n}\right\}_{n \in N}$ that collapses the folded arcs in $R$ onto the initial arcs of $S\left\{k_{n}, l_{n}\right\}_{n \in N}$.

Define

$$
\mathcal{M}_{0}=S^{o}\{2 n-1,2 n\}_{n \in N} \cup D \cup S\left\{k_{n}, l_{n}\right\}_{n \in N} \cup R .
$$

For each $p \in \mathcal{M}_{0}$, let

$$
\rho(p)= \begin{cases}h_{0}\left(\rho_{1}\left(\rho_{2}(p)\right)\right) & \text { if } p \in R \cup S\left\{k_{n}, l_{n}\right\}_{n \in N}, \\ p & \text { if } p \in D \cup S^{o}\{2 n-1,2 n\}_{n \in N} .\end{cases}
$$

By Propositions 3.5 and 3.6, $\rho$ is a retraction of $\mathcal{M}_{0}$ onto the continuum $D \cup$ $S^{o}\{2 n-1,2 n\}_{n \in N}$. By Propositions 3.2 and 3.5, D $\cup S^{o}\{2 n-1,2 n\}_{n \in N}$ does not have the fixed-point property. Therefore $\mathcal{M}_{0}$ does not have the fixed-point property.

Obviously, $\mathcal{M}_{0}$ is rational. Also, $\mathcal{M}_{0}$ is irreducible between the origin $\left(\frac{3}{2}, 0\right)$ of $S^{o}\{2 n-1,2 n\}_{n \in N}$ and the origin $(4,0)$ of $R$, since $\mathcal{M}_{o}=C l R \cup C l S^{o}\{2 n-1,2 n\}_{n \in N}$ and the sets $S^{o}\{2 n-1,2 n\}_{n \in N}, D, S\left\{k_{n}, l_{n}\right\}_{n \in N}$, and $R$ are pairwise disjoint.

We will prove in Section 5 that the unique non-degenerate tranche $D \cup$ $S\left\{k_{n}, l_{n}\right\}_{n \in N}$ of $\mathcal{M}_{0}$ has the fixed-point property.

Remark 4.1. Similar to the continuum $\mathcal{M}$ in $E^{3}$ defined in $[\mathrm{H}]$, the continuum $\mathcal{M}_{0}$ can be used to define another rational irreducible continuum in $E^{2}$ that admits a fixed-point-free surjection with the condition that all of its tranches have the fixed-point property.

\section{Proof of Theorem 3.3}

We will consider the case when the sequence $\left\{\frac{l_{n}-k_{n}}{k_{n}}\right\}_{n \in N}$ is unbounded.

Denoting the spiral $S\left\{k_{n}, l_{n}\right\}_{n \in N}$ by $S$, we assume there is a map $f$ of $D \cup S$ into itself that moves each point.

Then

(5.1). $f(S) \subset S$ and $p<_{S} f(p)$ for each $p \in S$ and $f(D)=D$ [M2, Prop. 3.2].

Hence, by [M2, Prop. 3.1],

(5.2). $f(A)=\alpha(A)$ and $f(\alpha(A))=A$, and 
(5.3). $f(L(A))=L(\alpha(A))$ and $f(L(\alpha(A)))=L(A)$.

Since $E_{-}^{2}$ is a neighborhood of $L(\alpha(A))$, it follows from the first equality in (5.3) that there is a neighborhood $P$ of $L(A)$ such that $f(P \cap S) \subset E_{-}^{2} \cap S$. We can assume $P$ is a closed rectangle lying in $E_{+}^{2}$ with sides perpendicular to the $x$-axis. By the second equality in (5.3), there is also a closed rectangular neighborhood $Q$ of $L(\alpha(A))$ such that $f(Q \cap S) \subset P \cap S$ and $Q \subset E_{-}^{2}$ and the sides of $Q$ are perpendicular to the $x$-axis. We can also assume that $P \cap(A \backslash \Gamma)=\emptyset=$ $Q \cap(\alpha(A) \backslash \alpha(\Gamma))$.

Let $n_{0}$ be an integer such that for each $n \geq n_{0}$ the sets $B_{n}\left(k_{n}\right) \cap P$ and $E_{n}\left(l_{n}\right) \cap Q$ are arcs. For each $n \geq n_{0}$, let $b_{n}$ denote the first point with respect to the order $<_{S}$ of $B_{n}\left(k_{n}\right) \cap P$ and let $e_{n}$ denote the first point of $E_{n}\left(l_{n}\right) \cap Q$.

For each $n>n_{0}$, define arcs

$$
I_{n}=S\left[\left(-\mu_{l_{n-1}},-3-(n-1)^{-1}\right), b_{n}\right] \text { and } J_{n}=S\left[\left(\mu_{k_{n}}, 3+n^{-1}\right), e_{n}\right] .
$$

Note that the sequences $\left\{I_{n}\right\}_{n>n_{0}}$ and $\left\{J_{n}\right\}_{n>n_{0}}$ converge to the arcs $A[(0,-3)$, $\left.\lim b_{n}\right]$ and $\alpha(A)\left[(0,3), \lim e_{n}\right]$, respectively.

Setting $E_{r}^{2}=\left\{(x, y) \in E^{2} \mid x \geq 0\right\}$ and $E_{l}^{2}=\left\{(x, y) \in E^{2} \mid x \leq 0\right\}$, there is an integer $n_{1} \geq n_{0}$ such that

(5.4). $f\left(I_{n}\right) \subset E_{l}^{2} \cup P$ and $f\left(J_{n}\right) \subset E_{r}^{2} \cup Q$ for each $n>n_{1}$.

This follows from (5.2) and the fact that $E_{l}^{2} \cup P$ and $E_{r}^{2} \cup Q$ are neighborhoods of $f\left(\lim I_{n}\right)=\lim f\left(I_{n}\right)$ and $f\left(\lim J_{n}\right)=\lim f\left(J_{n}\right)$, respectively.

For each $n>n_{1}$, let $H_{n}=P \cap \Gamma_{n}\left(k_{n}, l_{n}\right)$ and $K_{n}=E_{-}^{2} \cap \Gamma_{n}\left(k_{n}, l_{n}\right)$. Observe that $H_{n}$ and $K_{n}$ are arc components of $P \cap S$ and $E_{-}^{2} \cap S$, respectively, and $b_{n}$ is the end point of the arc $H_{n}$ that belongs to $E_{r}^{2}$, and $e_{n}$ is the end point of the arc $K_{n} \cap Q$ that belongs to $E_{l}^{2}$. Since $f(P \cap S) \subset E_{-}^{2} \cap S$, for each $n>n_{1}$ there is exactly one $i_{n} \in N$ such that $f\left(H_{n}\right) \subset K_{i_{n}}$. By (5.1), $i_{n} \geq n$.

We are going to prove

(5.5). $E_{i_{n}}\left(l_{i_{n}}\right) \subset f\left(I_{n} \cup B_{n}\left(k_{n}\right) \cup J_{n}\right)$ for each $n>n_{1}$.

The $\operatorname{arcs} I_{n}$ and $J_{n}$ both meet $H_{n}$, since $b_{n} \in H_{n} \cap I_{n}$ and $\left(\mu_{k_{n}}, 3+n^{-1}\right) \in H_{n} \cap J_{n}$, and therefore

$$
f\left(I_{n}\right) \cap K_{i_{n}} \neq \emptyset \neq f\left(J_{n}\right) \cap K_{i_{n}} \text { for each } n>n_{1} .
$$

Also, we have $f\left(I_{n}\right) \cap P \neq \emptyset \neq f\left(J_{n}\right) \cap P$ for each $n>n_{1}$, because $\left(-\mu_{l_{n-1}},-3-\right.$ $\left.(n-1)^{-1}\right) \in Q \cap I_{n}$ implies $f\left(\left(-\mu_{l_{n-1}},-3-(n-1)^{-1}\right)\right) \in f\left(I_{n}\right) \cap P$ and $e_{n} \in Q \cap J_{n}$ implies $f\left(e_{n}\right) \in f\left(J_{n}\right) \cap P$. Thus $f\left(I_{n}\right)$ meets an arc component $H_{m}$ of $P \cap S$ and $f\left(J_{n}\right)$ meets an arc component $H_{l}$ of $P \cap S$.

For each $n>n_{1}$,

(5.6). $f\left(I_{n}\right) \cap H_{m} \neq \emptyset$ implies $m=i_{n}$ and $f\left(J_{n}\right) \cap H_{l} \neq \emptyset$ implies $l=i_{n}+1$.

To establish (5.6), we denote $\Gamma_{n}\left(k_{n}, l_{n}\right)$ by $\Gamma_{n}$ and first observe that if $n<n^{\prime}$, then $p<_{S} q$ for each $p \in \Gamma_{n}$ and $q \in \Gamma_{n^{\prime}}$ such that $p \neq q$. Also, the order $<_{S}$ agrees with the counterclockwise orientation of $E^{2}$.

If $m<i_{n}$, then $f\left(I_{n}\right)$, going from $K_{i_{n}}$ to $H_{m}$ (clockwise), must contain $\Gamma_{i_{n}} \cap E_{+}^{2}$, and if $i_{n}<m$, then $f\left(I_{n}\right)$ goes from $K_{i_{n}}$ to $H_{m}$ counterclockwise so that $\left(\Gamma_{i_{n}+1} \cap\right.$ $\left.E_{+}^{2} \cap E_{r}^{2}\right) \backslash P \subset f\left(I_{n}\right)$; in both cases we have a contradiction of the first inclusion in (5.4). 
If $i_{n}+1<l$, then $f\left(J_{n}\right)$ goes from $K_{i_{n}}$ to $H_{l}$ counterclockwise so that $\Gamma_{i_{n}+1} \subset$ $f\left(J_{n}\right)$, and if $l<i_{n}+1$, then going clockwise we obtain $\Gamma_{i_{n}} \cap E_{+}^{2} \subset f\left(J_{n}\right)$; in both cases we have a contradiction of the second inclusion in (5.4). Hence (5.6) is true.

Thus

$$
f\left(I_{n}\right) \cap H_{i_{n}} \neq \emptyset \neq f\left(J_{n}\right) \cap H_{i_{n}+1} \text { for each } n>n_{1} .
$$

In view of (5.4), the inequalities $f\left(I_{n}\right) \cap H_{i_{n}} \neq \emptyset \neq f\left(I_{n}\right) \cap K_{i_{n}}$ imply that $\left(-2-i_{n}^{-1}, 0\right) \in f\left(I_{n}\right)$ and the inequalities $f\left(J_{n}\right) \cap K_{i_{n}} \neq \emptyset \neq f\left(J_{n}\right) \cap H_{i_{n}+1}$ imply that $\left(2+\left(i_{n}+1\right)^{-1}, 0\right) \in f\left(J_{n}\right)$. Thus both end points of the open arc $K_{i_{n}}$ belong to the image $f\left(I_{n} \cup B_{n}\left(k_{n}\right) \cup J_{n}\right)$ in $S$. Since $b_{n} \in I_{n} \cap B_{n}\left(k_{n}\right)$ and $\left(\mu_{k_{n}}, 3+n^{-1}\right) \in J_{n} \cap B_{n}\left(k_{n}\right)$, it follows that $I_{n} \cup B_{n}\left(k_{n}\right) \cup J_{n}$ is an arc in $S$ (with end points $\left(-\mu_{l_{n-1}},-3-(n-1)^{-1}\right)$ and $\left.e_{n}\right)$, and thus the image $f\left(I_{n} \cup B_{n}\left(k_{n}\right) \cup J_{n}\right)$ is an arc in $S$. Hence $K_{i_{n}} \subset f\left(I_{n} \cup B_{n}\left(k_{n}\right) \cup J_{n}\right)$. But, obviously, we have $E_{i_{n}}\left(l_{i_{n}}\right) \subset K_{i_{n}}$, which proves (5.5).

Claim 5.7. There exists a sequence $\left\{L_{n}\right\}_{n>n_{1}}$ of $\operatorname{arcs}$ in $S$ and a sequence $\left\{w_{n}\right\}_{n>n_{1}}$ of nonnegative integers such that

(i) each $L_{n}$ lies in $I_{n} \cup J_{n}$ or is a dip in $B_{n}\left(k_{n}\right)$,

(ii) each $f\left(L_{n}\right)$ contains $w_{n}$ humps of $E_{i_{n}}\left(l_{i_{n}}\right)$,

(iii) the sequence $\left\{L_{n}\right\}_{n>n_{1}}$ converges to an $\operatorname{arc} \lim L_{n} \subset D$, and

(iv) the sequence $\left\{w_{n}\right\}_{n>n_{1}}$ is unbounded.

To prove Claim 5.7, for each $n>n_{1}$, let $u_{n}$ denote the number of humps of $E_{i_{n}}\left(l_{i_{n}}\right)$ that are contained in the image $f\left(I_{n} \cup J_{n}\right)=f\left(I_{n}\right) \cup f\left(J_{n}\right)$.

Case 1. Suppose the sequence $\left\{u_{n}\right\}_{n>n_{1}}$ is unbounded. Then for each $n>n_{1}$, let $L_{n}$ be one of the $\operatorname{arcs} I_{n}$ or $J_{n}$, the image of which contains $w_{n} \leq u_{n}$ humps of $E_{i_{n}}\left(l_{i_{n}}\right)$ in such a way that the sequence $\left\{w_{n}\right\}_{n>n_{1}}$ is unbounded.

Case 2. Suppose $\left\{u_{n}\right\}_{n>n_{1}}$ is bounded. Let $n>n_{1}$. The number of humps of $E_{i_{n}}\left(l_{i_{n}}\right)$ is $l_{i_{n}}$, and the number of humps of $E_{i_{n}}\left(l_{i_{n}}\right)$ that are contained in $f\left(I_{n}\right) \cup$ $f\left(J_{n}\right)$ is $u_{n}$. By $(5.4), f\left(I_{n}\right)$ may contain a left subarc and $f\left(J_{n}\right)$ may contain a right subarc of $E_{i_{n}}\left(l_{i_{n}}\right)$. Therefore, by (5.5), $f\left(B_{n}\left(k_{n}\right)\right)$ contains at least $l_{i_{n}}-\left(u_{n}+2\right)$ humps of $E_{i_{n}}\left(l_{i_{n}}\right)$. Since $i_{n} \geq n$, it follows from (3.1) that $l_{i_{n}} \geq l_{n}$. Thus $l_{i_{n}}-$ $\left(u_{n}+2\right) \geq l_{n}-\left(u_{n}+2\right)$. Setting $s_{n}=\frac{l_{n}-k_{n}}{k_{n}}$, we have

$$
l_{n}-\left(u_{n}+2\right)=k_{n} s_{n}+k_{n}-\left(u_{n}+2\right) \text {. }
$$

However $k_{n} \rightarrow \infty$ and, in the case being considered, the sequence $\left\{u_{n}+2\right\}_{n>n_{1}}$ is bounded. Hence there exists an integer $n_{2} \geq n_{1}$ such that $k_{n}-\left(u_{n}+2\right) \geq 0$ for each $n>n_{2}$. It follows that $l_{n}-\left(u_{n}+2\right) \geq k_{n} s_{n}$ for each $n>n_{2}$. Thus, for each $n>n_{2}$, the series of $k_{n}$ dips of $B_{n}\left(k_{n}\right)$ is mapped by $f$ on a series of $k_{n} v_{n}$ consecutive humps of $E_{i_{n}}\left(l_{i_{n}}\right)$, where $v_{n}$ is the integer part of $s_{n}$.

Applying Lemma 2.1 (with $v=\mu=k_{n}$ ) we obtain a dip $V_{n}$ of $B_{n}\left(k_{n}\right)$ that is mapped on a series of $v_{n}$ consecutive humps of $E_{i_{n}}\left(l_{i_{n}}\right)$. Let $\left\{V_{m_{n}}\right\}_{n>n_{2}}$ be a convergent subsequence of $\left\{V_{n}\right\}_{n>n_{2}}$ such that $v_{m_{n}} \rightarrow \infty$. For each $n>n_{2}$, let $L_{n}=V_{m_{n}}$ and $w_{n}=v_{m_{n}}$. Let $L_{n}=I_{n}$ and $w_{n}=0$ whenever $n_{1} \leq n \leq n_{2}$. Note that $\lim L_{n}$ is either a dip in $\Gamma$ or the interval $\overline{(0,1)(0,3)}=L(\Gamma)=L(A)$. This concludes the proof of Claim 5.7.

Let $\left\{L_{n}\right\}_{n>n_{1}}$ and $\left\{w_{n}\right\}_{n>n_{1}}$ be sequences that satisfy conditions (i) - (iv) of Claim 5.7. For each $n>n_{1}$, let $\mathcal{P}_{w_{n}}\left(L_{n}\right)$ be the partition of the arc $L_{n}$ into $w_{n}$ consecutive subarcs with the same length. By (iv), the sequence $\left\{w_{n}\right\}_{n>n_{1}}$ is unbounded. Instead of taking a strictly increasing subsequence of $\left\{w_{n}\right\}_{n>n_{1}}$, we assume that $\left\{w_{n}\right\}_{n>n_{1}}$ itself is strictly increasing. Hence $w_{n} \rightarrow \infty$. 
By (iii), the sequence $\left\{\mathcal{P}_{w_{n}}\left(L_{n}\right)\right\}_{n>n_{1}}$ of partitions converges to a decomposition $\mathcal{P}$ of the $\operatorname{arc} \lim L_{n} \subset D$. Since $w_{n} \rightarrow \infty$, it follows from (i) that each element of $\mathcal{P}$ consists of a single point. By (ii), each $f\left(L_{n}\right)$ contains $w_{n}$ humps of $E_{i_{n}}\left(l_{i_{n}}\right)$. For each $n>n_{1}$, it follows from Lemma 2.1 (with $v=\mu=w_{n}$ ) that the partition $\mathcal{P}_{w_{n}}\left(L_{n}\right)$ has an element $L_{j_{n}, n}$, the image of which contains an entire hump of $E_{i_{n}}\left(l_{i_{n}}\right)$. Passing to the limit with a convergent subsequence of the sequence $\left\{L_{j_{n}, n}\right\}_{n>n_{1}}$, we obtain a point (of the limit arc $\lim L_{n}$ ), the image of which contains the limit of the corresponding humps, i.e., either a hump of $\alpha(A)$ or the interval $L(\alpha(A))$, a contradiction.

By a dual argument, the assumption that $\left\{\frac{k_{n+1}-l_{n}}{l_{n}}\right\}_{n \in N}$ is unbounded also leads to a contradiction.

\section{Related Questions}

The tranche $D \cup S\left\{k_{n}, l_{n}\right\}_{n \in N}$ of $\mathcal{M}_{0}$ separates $E^{2}$.

Question 6.1. Must a plane continuum of type $\lambda$ have the fixed-point property if none of its tranches separates the plane?

A plane continuum of type $\lambda$ separates the plane if and only if at least one of its tranches separates the plane $[\mathrm{Mr}]$. Since it is not known if every nonseparating plane continuum has the fixed-point property [Bi, Ques. 3], [K-W, p. 66, p. 145], Question 6.1 should also be considered with the additional assumption that each tranche has the fixed-point property. A counterexample must contain an invariant indecomposable continuum in one of its tranches $[\mathrm{B}],[\mathrm{I}], \mathrm{S}]$.

Question 6.2. Must a plane continuum of type $\lambda$ have the fixed-point property if each of its tranches has the fixed-point property and its decomposition is continuous?

A counterexample to Question 6.2 cannot be rational since it must have an indecomposable tranche $[\mathrm{D}]$.

\section{REFERENCES}

[A] M. M. Awartani, The fixed remainder property for self-homeomorphisms of Elsa continua, Topology Proc. 11 (1986), 225-238. MR 89g:54073

[B] H. Bell, On fixed point properties of plane continua, Trans. Amer. Math. Soc. 128 (1967), 539-548. MR 35:4888

[Bi] R. H. Bing, The elusive fixed point property, Amer. Math. Monthly 76 (1969), 119-132. MR 38:5201

[D] E. Dyer, Irreducibility of the sum of the elements of a continuous collection of continua, Duke Math. J. 20 (1953), 589-592. MR 15:335f

$[\mathrm{H}] \quad$ C. L. Hagopian, Irreducible continua without the fixed-point property, Bull. Pol. Acad. Sci. Math. 51 (2003), 121-127.

[H-M] C. L. Hagopian and R. Mańka, Simple spirals on double Warsaw circles, Topology and its Appl. 128 (2003), 93-101. MR 2004c:54029]

[I] S. Iliadis, Positions of continua on the plane and fixed points, Vestnik Moskov. Univ. Ser. I Mat. Mekh. 1970, no. 4, 66-70. MR 44:4726

$[\mathrm{K}-\mathrm{W}]$ V. Klee and S. Wagon, Old and New Unsolved Problems in Plane Geometry and Number Theory, Dolciani Mathematical Expositions, vol. 11, Math. Assoc. Amer., Washington, DC, 1991. MR 92k:00014

[Ku1] C. Kuratowski, Théorie des continus irréductibles entre deux points II, Fund. Math. 10 (1927), 225-276.

[Ku2] , Topology, Vol. 2, 3rd ed., Monografie Mat., Tom 21, PWN, Warsaw, 1961; English transl., Academic Press, New York; PWN, Warsaw, 1968. MR 41:4467 
[L] I. W. Lewis, Continuum theory problems, Topology Proc. 8 (1983), 361-394. MR 86a:54038

[M1] R. Mańka, On irreducibility and indecomposability of continua, Fund. Math. 129 (1988), 121-131. MR 89g:54079

[M2] On spirals and the fixed point property, Fund. Math. 144 (1994), 1-9. MR 95c:54061

[Mr] R. L. Moore, Concerning upper semi-continuous collections of continua, Trans. Amer. Math. Soc. 27 (1925), 416-428.

[N] S. B. Nadler, Continua which are a one-to-one continuous image of $[0, \infty)$, Fund. Math. 75 (1972), 123-133. MR 47:5848

[S] K. Sieklucki, On a class of plane acyclic continua with the fixed point property, Fund. Math. 63 (1968), 257-278. MR 39:2139

Department of Mathematics, California State University, Sacramento, Sacramento, CALifornia 95819-6051

E-mail address: hagopian@csus.edu

Institute of Mathematics, Polish Academy of Sciences, Śniadeckich 8, 00-956 Warsaw, POLAND

E-mail address: manka@impan.gov.p1 